\section{(6) OPEN ACCESS}

\title{
Mesenchymal stem cells: mechanisms and role in bone regeneration
}

\author{
Yunhao Qin, Junjie Guan, Changqing Zhang
}

Shanghai Sixth People's Hospital affiliated to Department of Orthopaedic, Shanghai Jiaotong University, Shanghai, China

\section{Correspondence to} Dr Changqing Zhang, Shanghai No. 6 People's Hospital Affiliated to Shanghai Jiaotong University, Shanghai 200030, China; zhangcq@sjtu. edu.cn

Received 20 September 2013 Revised 29 September 2014 Accepted 30 September 2014
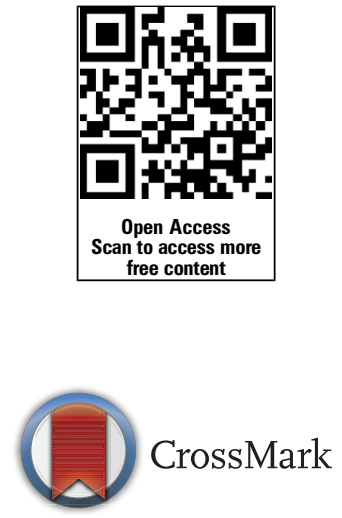

To cite: Qin Y, Guan J, Zhang C. Postgrad Med J 2014;90:643-647.

\begin{abstract}
Stimulating bone growth and regeneration, especially in patients with delayed union or non-union of bone, is a challenge for orthopaedic surgeons. Treatments employed for bone regeneration are based on the use of cells, biomaterials and factors. Among these therapies, cell treatment with mesenchymal stem cells (MSCs) has a number of advantages as MSCs: (1) are multipotent cells that can migrate to sites of injury; (2) are capable of suppressing the local immune response; and (3) are available in large quantities from the patients themselves. MSC therapies have been used for stimulating bone regeneration in animal models and in patients. Methods of application range from direct MSC injection, seeding MSCs on synthetic scaffolds, the use of gene-modified MSCs, and hetero-MSCs application. However, only a small number of these cell-based strategies are in clinical use, and none of these treatments has become the gold standard treatment for delayed or non-union of bone.
\end{abstract}

\section{INTRODUCTION}

Our understanding of mesenchymal stem cells (MSCs) is based on work carried out in the 1960s by Friedenstein and coworkers ${ }^{1}$ who observed that the bone marrow is a source of stem cells for mesenchymal tissue. They observed that these cells are plastic-adherent, fibroblast-like cells that can differentiate into chondrocytes, adipocytes and osteoblasts. Much research has shown that MSC treatment may be very useful for bone repair. The treatment offers the advantages but not the disadvantages of embryonic stem cells (ESCs): MSC use presents less risk of malignant disease development and avoids the ethical problems associated with ESCs. Although great success has been achieved with MSCs in promoting bone regeneration in animal models, there are a number of gaps in our knowledge which need to be addressed before this treatment can be widely applied in clinical practice. For example, the mechanism by which MSCs stimulate and mediate bone regeneration in vivo is unknown. Also, the relatively few in vivo experiments require in vitro expansion; however, it is difficult to maintain a stable phenotype in vitro.

\section{FEATURES OF MSCS}

MSCs are derived from a number of sources and act as immune suppressors and endocrine secretors, thereby exerting their regenerative effect.

Most parts of the human body including the bone marrow, adipose tissue, skin, liver and placenta produce MSCs. However, it is important to identify their source as MSCs from different origins have different potential to differentiate into various cell lineages. This can be done by identifying cluster of differentiation (CD) markers, since MSCs from different sources have distinct CD markers; for example, the typical CD markers of MSCs from non-haematopoietic tissue are CD34-, CD11a-, CD45- and CD19-, while the typical CD markers of bone-derived MSCs are CD105, CD73, CD90, CD45-, CD34- and HLA-DR-. ${ }^{2}$ In addition to identifying their origin, the $C D$ markers also indicate MSC function and features. According to research by Yannarelli et al, ${ }^{3}$ CD146 MSCs are vigorous and can self-renew: after gene expression of CD146 is downregulated or silenced, this self-renewal ability is weakened or disappears.

The most important feature of MSCs is that, under certain in vitro conditions, the cells are capable of differentiating into mesodermal cells such as osteoblasts, adipose cells, cartilage cells ${ }^{4}$ or skeletal muscle cells. ${ }^{5}$ This process of differentiation is a basic step in promoting regeneration. In addition, research has shown that bone regeneration can be promoted by adding dexamethasone, ascorbic acid and $\beta$-glycerophosphate to the culture medium, while the addition of dexamethasone, insulin and rosiglitazone in the medium induces MSCs to differentiate into adipose cells.

MSCs inhibit the immune response in two ways: by the contact immune response ${ }^{6}$ and by the noncontact immune response. ${ }^{7}$ Bartholomew et $a l^{8}$ found that, through the contact immune response, MSCs could inhibit the immune response of lymphocytes, prevent the maturation of antigenpresenting cells and weaken the immune function of natural killer cells. Since MSCs can act as immune suppressors, they have been used to treat several immune diseases. Le Blanc et $a l^{9}$ successfully used in vitro expanded MSCs to ameliorate severe graft versus host disease (GVHD). The median dose of MSCs used in their study was $1.4 \times 10^{6} / \mathrm{kg}$. Of the 55 patients treated, 27 received one dose, 22 received two doses, four received three doses, one received four doses and one received five doses. Thirty patients achieved a complete response and nine patients showed improvement. However, three patients developed recurrent malignant disease and one patient developed acute myeloid leukaemia. The study results showed that the 1-year mortality of patients treated with MSCs was lower than for patients not treated with MSCs (11 of 30 patients $(37 \%)$ died in the MSC treatment group compared with 18 of 25 patients $(72 \%)$ in the non-MSC treatment group). Furthermore, the 2-year survival rate of patients in the MSC treatment group was higher than in the non-MSC treatment group (16 of 30 patients $(53 \%)$ in the MSC treatment group survived 
compared with four of 25 patients (16\%) in the non-MSC treatment group).

A new theory that MSCs may act as endocrine secretors has recently attracted the attention of researchers. For instance, when placed in a hypoxic environment, Toll-like receptor ligands and trauma site inflammation factors induce MSCs to secrete a number of factors that stimulate tissue regeneration, including epidermal growth factor, insulin-like growth factor and fibroblast growth factor, which may significantly upregulate and promote angiogenesis and inhibit apoptosis. Some precursors have already been found to support this hypothesis. $\mathrm{Xu}$ et $a l^{10}$ showed that MSCs could inhibit apoptosis of lymphocytes through the contact immune response. The inhibitory effect was positively correlated with the number of MSCs. In addition, the rate of apoptosis of lymphocytes in an experimental group in which interleukin 6 (IL-6) antibodies were added to the co-culture environment was higher than in the control group treated with MSCs only. This result showed that, by neutralising IL-6, the immune inhibition effect of MSCs was weakened. In other words, the contact immune response stimulated the expression and secretion of IL- 6 in MSCs, thus inhibiting lymphocyte apoptosis.

In summary, the large number of MSCs produced by the body together with their pluripotency ensures the availability of different types of cells that help to promote regeneration, and their immune inhibitory effect makes transplantation much safer. These unique features mean that MSCs have a significant role in stimulating bone regeneration.

\section{INFLUENCES ON THE DIFFERENTIATION OF MSCS}

Target cell lineage differentiation is critical for the regenerative effect of MSCs, and part of the mechanism of this process has now been elucidated. The microenvironment has the strongest influence on the maturation and differentiation of MSCs, but cell-to-cell communication, physical factors and cell structure have also been found to have an effect. However, the mechanisms by which these factors affect MSCs are still not fully understood.

The microenvironment plays a key role in controlling MSC differentiation. The process is influenced by the presence of different proteins, hormones and growth factors. Bone morphogenetic protein (BMP) is the classic osteogenic protein, ${ }^{11}$ and Liu et $a l^{12}$ have elucidated the exact mechanism by which BMP affects MSCs. This protein generates the transcription factor Smads by combining with type I or II serine/threonine receptor on the MSC membrane to activate 203 gene loci which control the osteoblast differentiation of MSCs. Furthermore, Mizrahi et $a l^{13}$ showed that BMP-6 was a stronger bone induction factor than BMP-2. Various hormones have also been shown to affect MSCs including estrogen, the mechanism and effect of which have been elucidated. ${ }^{14}$ Auld et $a l^{15}$ showed that estrogen can bind and activate the estrogen receptor (ER) to regulate expression of downstream genes to perform cellular functions. In particular, the orphan nuclear receptor estrogen-related receptor $\alpha$ $(\mathrm{ESR} \alpha)$ has been shown to bind with estrogen and activate the Wnt/ß-catenin pathway to promote MSC osteogenic differentiation. The effect of small molecules and the interior milieu is now being extensively studied, especially hypoxic environments. Kim $e t ~ a l^{16}$ found that a change in oxygen pressure could influence the expression of Runx-2 and peroxisome proliferation activated receptor $\gamma(\mathrm{PPAR}-\gamma)$ and so control MSC differentiation.

Cell-to-cell communication, especially as regards receptors on the cell surface, also affects MSCs. Migration to the bone surface is a key step in the maturation of bone lineage MSCs. Transmembrane integrin receptors recognise ligands which mediate cell matrix and cell-to-cell interaction and induce MSC maturation and attachment to the bone surface. Once this process is complete, the MSCs immediately start to secrete various proteins (such as bone glaprotein (BGP), osteopontin, type I collagen and fibronectin) to establish a chemical gradient, which may guide further MSCs to attach to the bone surface. Guan et $a^{17}$ added synthetic specific peptidomimetic ligands (LLP2A) to MSCs. These polypeptides, which recognised integrin $\alpha 4 \beta 1$ receptors on the MSC surface, were combined with alendronate which has a high affinity for bone. Thus, LLP2A-modified MSCs were directed to the bone surface and started to induce bone formation and regeneration.

Differentiation is also influenced by physical factors. Yue et $a l^{18}$ tested the osteogenic effect of ultrasound. Polymerase chain reaction of Runx-2 showed that MSCs cultured for $10 \mathrm{~min} /$ day in an osteogenic medium with $1 \mathrm{kHz}$ ultrasound had much higher expression of osteogenic protein than MSCs cultured in the medium without ultrasound. Liu et $a l^{19}$ found that fluid shear stress might activate MAPK, NO/cGMP/PKG and the $\mathrm{Ca}^{2+}$ pathway to promote the osteogenic differentiation of MSCs.

Preliminary findings suggest that the cell structure may have an effect on MSC differentiation. During the different stages of MSC osteogenic differentiation, the actin cytoskeleton ${ }^{20}$ changes from thick actin stress fibres to dispersed actin cytoskeleton. If the process is disrupted by cytochalasin, the Rock/RhoA pathways are inhibited, which usually results in differentiation of MSCs into adipose tissue.

In summary, the regenerative effect of MSCs is mediated by various factors. However, these mechanisms are based on in vitro observations and the in vivo situation is much more complicated. Much research is required before the differentiation and maturation of MSCs can be precisely controlled in vivo.

\section{APPLICATION OF MSCS}

Several approaches to MSC treatment have been used to promote bone regeneration in both animal models and clinical practice. The most common MSC treatment is direct injection. Some studies have also shown that attaching MSCs on a bioscaffold is an effective way to treat non-union of bone. The effectiveness and safety of gene-modified and hetero-MSCs have also been explored in animal models.

\section{Direct injection of MSCs}

For direct injection of MSCs, bone marrow is usually aspirated from the iliac crest and injected at the site of bone non-union. The aspirated mixture contains many MSCs and is called bone marrow aspirate concentrate (BMAC).

The effectiveness of direct injection of MSCs has been shown in both animal experiments and clinical studies. A report by Lee et $a l^{21}$ described direct injection of MSCs with hyaluronic acid at defects on the medial femoral condyle in 27 pigs. The animals were killed 6 and 12 weeks later and results showed that the experimental group had cartilage regeneration while the control group demonstrated fibrous regeneration.

Results in animal models led to the use of MSCs to repair large bone defects in the clinical setting. In 1998, Connolly ${ }^{22}$ used direct injection of BMAC to heal non-union of the tibia in 20 patients, 18 of whom showed full recovery after 6-8 months. Other clinical studies also support the effectiveness of the direct injection of MSCs. Hernigou and Beaujean ${ }^{23}$ found a positive correlation between the quantity of BMAC administered by percutaneous injection and mineralised bone 
formation to treat non-union of the tibia, and reported good results in 60 patients.

Although direct injection is clearly effective in promoting bone regeneration, some drawbacks of this technique have been reported in animal studies. These include the development of local ischaemia following direct injection into an artery and micro embolisation in the lungs following intravenous injection. In a related experimental study, Janowski et $a l^{24}$ directly injected MSCs into the carotid artery of rats and found that an injection velocity higher than $0.2 \mathrm{~mL} / \mathrm{min}$ could cause local ischaemia; however, if fewer than $1 \times 10^{6}$ MSCs were used, the injection did not cause local ischaemia.

The effectiveness and safety of direct injection of MSCs in humans still needs further investigation and assessment.

\section{MSCs and biomaterial scaffolds}

Direct injection of MSCs has been found to be ineffective for very large bone defects or non-union of bone. However, such cases can be effectively treated by attaching MSCs to a biomaterial scaffold. The ideal biomaterial scaffold must be biodegradable, biocompatible, support colony formation and be a good bone inductor. Hydroxyapatite (HA) ${ }^{25}$ and tricalcium phosphate $(\mathrm{TCP})^{26}$ are commonly used in clinical practice to promote bone regeneration because of their good bone induction properties.

The effectiveness of bioscaffolds has been confirmed by many researchers. Morishita $e t a l^{27}$ treated defects after tumour resection at the distal tibia by attaching autologous MSCs to HA to induce bone regeneration. The results showed that two patients were able to weight bear on the affected extremities 2 weeks after transplantation and one patient was able to weight bear 3 weeks after transplantation. A CT scan at 3 weeks showed a gradual increase in the density of the transplantation area. Furthermore, several studies have also investigated the use of in vitro expanded MSCs for the regeneration of bone. In a phase I clinical trial, Quarto et $a l^{28}$ attached ex vivo expanded MSCs on $\mathrm{HA}$ to treat bone defects in three patients with successful results.

Nanomaterials are also being actively investigated for use as scaffolds for optimal outcomes in animal models. Fricain et al ${ }^{29}$ successfully used a hydrophilic polysaccharide scaffold with nano-HA to induce bone regeneration. In vitro experiments showed that MSCs in a culture environment with low bone induction factors and attached to this type of scaffold were still able to congregate and express bone-specific markers. Additionally, animal models showed that this scaffold successfully and independently induced calcification of the surrounding tissue and bone formation. Biomaterial scaffolds with MSCs have been fully developed and numerous successful treatments have been conducted by researchers and doctors in both animals and humans. Three-dimensional (3D) prints of scaffolds using technologies such as rapid prototyping have also been used for developing scaffolds to promote bone regeneration. Duarte Campos et $a l^{30}$ encapsulated MSCs into agarose hydrogels and made a 3D print, supported by fluorocarbon, and achieved good cell culture results.

However, more clinical trials are required to investigate the effectiveness, safety and side effects of the traditional method of attaching MSCs on biomaterials before it can be widely applied in clinical practice. Furthermore, it needs to be demonstrated that the new materials are superior to the more traditional ones.

\section{Gene-modified MSCs}

Delivering genes to enhance MSC function has been explored by many researchers, with BMP genes most commonly used to promote the bone formation activity of MSCs. ${ }^{31-34}$ Most gene-modified MSCs have been used in animal models.

The genetic technique can be utilised when MSCs are transplanted back into animals or patients after in vivo modification. Gamradt et $a l^{31}$ investigated 24 mice with severe combined immunodeficiency. The mice were divided into two groups (12 mice in each group) and one group was treated with adenovirus and BMP-2 gene-transfected MSCs while the other group was treated with normal MSCs. The MSCs were transplanted into $3 \mathrm{~mm}$ defects in the left forelimb of the mice. Three mice from each group were killed at 3, 7, 14 and 21 days. X-rays at 7 days showed that the experimental group achieved bone formation before the control group. In addition, bone formation was much more stable in the experimental group than in the control group at 21 days.

Another way to obtain gene-modified MSCs is to directly inject a viral vector to transfect the gene at sites of non-union of bone. Ishihara et $a l^{35}$ directly injected BMP-2 and BMP-6 transfected adenovirus at bone defects in horses to explore the regenerative effect. Radiographic assessment showed greater and earlier bone formation in the experimental group, while CT and biomechanical testing showed more mineralised callus and torsional strength. Histological evaluation showed that the bone defects in the experimental group had greater formation of mature cartilage and bone nodules.

The target gene can also be developed by using gene-activated matrix (GAM), which consists of collagenous scaffold with plasmid DNA encoding osteogenic products. When GAM is inserted into a bone lesion, host cells enter it, are transfected by the DNA and secrete the osteogenic gene product. The effectiveness of GAM in promoting bone regeneration has been investigated by Bonadio et al. ${ }^{36}$ GAM was implanted at sites of bone injury in dogs and the retention and expression of plasmid DNA were evaluated for at least 6 weeks. The results showed that the bone induction effect of GAM is stable, reproducible, dose-dependent and time-dependent.

Epigenetic modification and interference of RNA are major techniques commonly used to modify genes. Du et $a l^{37}$ showed in vivo that a widely present histone demethylase FBXL11 could demethylate the epiregulin gene to inhibit osteogenic differentiation of MSCs. Furthermore, Zeng et al ${ }^{38}$ found that miRNA-100 could bind to BMP-2 receptor and successfully inhibit osteogenic differentiation of adipose-derived MSCs.

Although promising results have been achieved in animal models, getting gene-modified MSCs into human clinical trials is a lengthy, expensive and frustrating process. Furthermore, safety is the top priority for any gene therapy, but safety testing requires pharmacology and toxicology studies and distribution analysis of the transferred genomes. In addition, it is complicated and expensive to develop clinical grade vectors. A further problem is how to develop noninvasive techniques to evaluate bone quality and mechanical strength.

\section{Future prospects}

Many researchers have studied the effects of MSCs in stimulating bone regeneration. Although results are very encouraging, 
many problems remain. First, most experiments have explored in vitro or animal mechanisms and there is little information about the in vivo mechanisms of MSCs. Second, although MSCs have been applied in clinical practice and have been used to treat hundreds of patients, their efficacy is still inferior to that of bone autografts. Third, further studies are required to obtain the most viable MSCs through cell culture and expansion. Fourth, the efficacy of MSCs of various origins also needs further research and assessment. With the development of biomaterials and molecular techniques, MSCs will become more important in the current search for bone regeneration therapies.

\section{Main messages}

- Mesenchymal stem cells (MSCs) are multi-potent cells that derive from the bone marrow, migrate to sites of injury, promote bone regeneration and can inhibit immune responses.

- In vitro, MSCs can differentiate into mesodermal cellsosteoblasts, adipose cells and cartilage cells.

- MSCs have therapeutic potential and offer an approach for promoting bone regeneration and improving the efficacy of transplantation.

\section{Current research questions}

- A better understanding of the in vivo mechanisms of action of mesenchymal stem cells (MSCs) is required.

- Methods to achieve viable quantities of MSCs through cell culture and expansion need to be developed.

- The efficacy of MSCs from different sources needs to be ascertained.

\section{Key references}

- Guan M, Yao W, Liu R, et al. Directing mesenchymal stem cells to bone to augment bone formation and increase bone mass. Nat Med 2012;18:456-62.

- Yannarelli G, Pacienza N, Cuniberti L, et al. Brief report: The potential role of epigenetics on multipotent cell differentiation capacity of mesenchymal stromal cells. Stem Cells 2013;31:215-20.

- Du J, Ma Y, Ma P, Wang S, et al. Demethylation of epiregulin gene by histone demethylase FBXL11 and BCL6 corepressor inhibits osteo/dentinogenic differentiation. Stem Cells 2013;31:126-136.

- Schubert T, Poilvache H, Galli C, et al. Galactosyl-knock-out engineered pig as a xenogenic donor source of adipose MSCs for bone regeneration. Biomaterials 2013;34:3279-89.

- Yue Y, Yang X, Wei X, et al. Osteogenic differentiation of adipose-derived stem cells prompted by low-intensity pulsed ultrasound. Cell Prolif 2013;46:320-7.

\section{Self assessment questions}

1. Which markers are typical of bone derived mesenchymal stem cells?
A. CD105, CD73, CD90
B. CD45-, CD34-, HLA-DR-
C. CD45, CD34, CD11-

2. Which factors induce osteogenic differentiation by mesenchymal stem cells?
A. Bone morphogenetic protein (BMP)
B. Estrogen
C. Vascular endothelial growth factor (VEGF)

3. Which biomaterials are commonly used in clinic practice?
A. Hydroxyapatite (HA)
B. Tricalcium phosphate (TCP)
C. LLP2A

4. Which obstacles prevent the wide application of MSCs in clinic practice?
A. Treatment efficacy has not been proven.
B. There are ethical problems with using MSCs.
C. The safety of MSCs needs further investigation.

5. Which are special features of MSCs?
A. MSCs can suppress the immune response.
B. MSCs can promote trauma regeneration.
C. MSCs can differentiate into adipose tissue.

Contributors $Y Q$ designed and wrote the manuscript. JG and CZ provided essential advice and revised the manuscript.

\section{Competing interests None.}

Provenance and peer review Not commissioned; externally peer reviewed.

Open Access This is an Open Access article distributed in accordance with the Creative Commons Attribution Non Commercial (CC BY-NC 3.0) license, which permits others to distribute, remix, adapt, build upon this work non-commercially, and license their derivative works on different terms, provided the original work is properly cited and the use is non-commercial. See: http://creativecommons.org/ licenses/by-nc/3.0/

\section{REFERENCES}

1 Afanasyev BV, Elstner EE, Zander AR. A. J. Friedenstein, founder of the mesenchymal stem cell concept. Cell Ther Transplant 2009;1:35-8.

2 Hagmann S, Moradi B, Frank S, et al. Different culture media affect growth characteristics, surface marker distribution and chondrogenic differentiation of human bone marrow derived mesenchymal stromal cells. BMC Musculoskelet Disord 2013;30:223.

3 Yannarelli G, Pacienza N, Cuniberti L, et al. Brief report: The potential role of epigenetics on multipotent cell differentiation capacity of mesenchymal stromal cells. Stem Cells 2013:31:215-20.

4 Moroni L, Fornasari PM. Human mesenchymal stem cells: a bank perspective on the isolation, characterization and potential of alternative sources for the regeneration of musculoskeletal tissues. J Cell Physiol 2013;228:680-7.

5 Wu L, Cai X, Zhang S, et al. Regeneration of articular cartilage by adipose tissue derived mesenchymal stem cells: perspective from stem cell biology and molecular medicine. J Cell Physiol 2013;228:938-44.

6 Sánchez-Abarca LI, Alvarez-Laderas I, Díez Campelo M, et al. Uptake and delivery of antigens by mesenchymal stem cells. Cytotherapy 2013;15:673-8.

7 Yagi H, Soto-Gutierrez A, Parekkadan B, et al. Mesenchymal stem cells: mechanisms of immunomodulation and homing. Cell Transplant 2010;19:667-79.

8 Bartholomew A, Polchert D, Szilagyi E, et al. Mesenchymal stem cells in the induction of transplantation tolerance. Transplantation 2009;87:55-7.

9 Le Blanc K, Frassoni F, Ball L, et al. Mesenchymal stem cells for treatment of steroid-resistant, severe, acute graft-versus-host disease: a phase II study. Lancet 2008;371:1579-86. 
10 Xu G, Zhang Y, Zhang L, et al. The role of IL-6 in inhibition of lymphocyte apoptosis by mesenchymal stem cells. Biochem Biophys Res Commun 2007:361:745-50.

11 Bai Y, Li P, Yin G, et al. BMP-2, VEGF and bFGF synergistically promote the osteogenic differentiation of rat bone marrow-derived mesenchymal stem cells. Biotechnol Lett 2013;35:301-8.

12 Liu $\mathrm{H}$, Peng $\mathrm{H}, \mathrm{Wu} \mathrm{Y}$, et al. The promotion of bone regeneration by nanofibrous hydroxyapatite/chitosan scaffolds by effects on integrin-BMP/Smad signaling pathway in BMSCs. Biomaterials 2013:34:4404-17.

13 Mizrahi O, Sheyn D, Tawackoli W, et al. BMP-6 is more efficient in bone formation than BMP-2 when overexpressed in mesenchymal stem cells. Gene Ther 2013;20:370-7.

14 Chen FP, Hu CH, Wang KC. Estrogen modulates osteogenic activity and estrogen receptor mRNA in mesenchymal stem cells of women. Climacteric 2013:16:154-60.

15 Auld KL, Berasi SP, Liu Y, et al. Estrogen-related receptor $\alpha$ regulates osteoblast differentiation via Wnt/B-catenin signaling. J Mol Endocrinol 2012;48:177-91.

$16 \operatorname{Kim}$ M, Kim C, Choi YS, et al. Age-related alteration in mesenchymal stem cell related to shift in differentiation from osteogenic to adipogenic potential: implication to age-associated bone diseases and defects. Mech Ageing Dev 2012;133:215-25.

17 Guan M, Yao W, Liu R, et al. Directing mesenchymal stem cells to bone to augment bone formation and increase bone mass. Nat Med 2012;18:456-62.

18 Yue $Y$, Yang $X$, Wei $X$, et al. Osteogenic differentiation of adipose-derived stem cells prompted by low-intensity pulsed ultrasound. Cell Prolif 2013:46:320-7.

19 Liu L, Yuan W, Wang J. Mechanisms for osteogenic differentiation of human mesenchymal stem cells induced by fluid shear stress. Biomech Model Mechanobiol 2010;9:659-70.

20 Mathieu PS, Loboa EG. Cytoskeletal and focal adhesion influences on mesenchymal stem cell shape, mechanical properties, and differentiation down osteogenic, adipogenic and chondrogenic pathways. Tissue Eng Part B Rev 2012;18:436-44.

21 Lee KB, Hui JH, Song IC. Injectable mesenchymal stem cell therapy for large cartilage defects - a porcine model. Stem Cells 2007;25:2964-71.

22 Connolly JF. Clinical use of marrow osteoprogenitor cells to stimulate osteogenesis. Clin Orthop Relat Res 1998;355(Suppl):S257-66.

23 Hernigou P, Beaujean F. Treatment of osteonecrosis with autologous bone marrow grafting. Clin Orthop Relat Res 2002;405:14-23.

24 Janowski M, Lyczek $A$, Engels $C$, et al. Cell size and velocity of injection are major determinants of the safety of intracarotid stem cell transplantation. J Cereb Blood Flow Metab 2013;33:921-7.

25 Liu P, Song J. Sulfobetaine as a zwitterionic mediator for 3D hydroxyapatite mineralization. Biomaterials 2013;34:2442-54

26 Wang W, Ma N, Kratz K, et al. The influence of polymer scaffolds on cellular behaviour of bone marrow derived human mesenchymal stem cells. Clin Hemorheol Microcirc 2012;52:357-73.

27 Morishita $\mathrm{T}$, Honoki $\mathrm{K}$, Ohgushi $\mathrm{H}$, et al. Tissue engineering approach to the treatment of bone tumors: three cases of cultured bone grafts derived from patients' mesenchymal stem cells. Artif Organs 2006;30:115-18.
28 Quarto R, Mastrogiacomo M, Cancedda R, et al. Repair of large bone defects with the use of autologous bone marrow stromal cells. N Engl J Med 2001;344:385-6.

29 Fricain JC, Schlaubitz S, Le Visage C, et al. A nano-hydroxyapatite-pullulan/ dextran polysaccharide composite macroporous material for bone tissue engineering. Biomaterials 2013:34:2947-59.

30 Duarte Campos DF, Blaeser A, Weber $M$, et al. Three-dimensional printing of stem cell-laden hydrogels submerged in a hydrophobic high-density fluid. Biofabrication 2013:5:015003.

31 Gamradt SC, Abe N, Bahamonde ME, et al. Tracking expression of virally mediated BMP-2 in gene therapy for bone repair. Clin Orthop Relat Res 2006;450:238-45.

32 Dwivedi PP, Anderson PJ, Powell BC, et al. Development of an efficient, non-viral transfection method for studying gene function and bone growth in human primary cranial suture mesenchymal cells reveals that the cells respond to BMP2 and BMP3. BMC Biotechnol 2012;12:45.

33 Zhang Y, Cheng N, Miron R, et al. Delivery of PDGF-B and BMP-7 by mesoporous bioglass/silk fibrin scaffolds for the repair of osteoporotic defects. Biomaterials 2012:33:6698-708.

34 Yuan J, Cui L, Zhang WJ, et al. Repair of canine mandibular bone defects with bone marrow stromal cells and porous beta-tricalcium phosphate. Biomaterials 2007:28:1005-13.

35 Ishihara A, Shields KM, Litsky AS, et al. Osteogenic gene regulation and relative acceleration of healing by adenoviral-mediated transfer of human BMP-2 or -6 in equine osteotomy and ostectomy models. J Orthop Res 2008;26:764-71.

36 Bonadio J, Smiley E, Patil P, et al. Direct plasmid gene delivery in vivo: prolonged therapy results in reproducible tissue regeneration. Nat Med 1999;5:753-9.

37 Du J, Ma Y, Ma P, et al. Demethylation of epiregulin gene by histone demethylase FBXL11 and BCL6 corepressor inhibits osteo/dentinogenic differentiation. Stem Cell 2013;31:126-36.

38 Zeng Y, Qu X, Li H, et al. MicroRNA-100 regulates osteogenic differentiation of human adipose-derived mesenchymal stem cells by targeting BMPR2. FEBS Lett 2012;586:2375-81.

\section{Answers}

1. (A) and (B)

2. (A) and (B).

3. (A) and (B).

4. (A), (B) and (C).

5. (A), (B) and (C). 